\title{
Two Simple Proofs of a Theorem by Harris*
}

\author{
Guilherme Carmona
}

October 3, 2005

\begin{abstract}
We present two simple proofs of existence of subgame perfect equilibria in continuous games with perfect information.

Journal of Economic Literature Classification Numbers: C73.
\end{abstract}

${ }^{*}$ I wish to thank Kemal Badur, and Jan Werner for very helpful comments. Financial support from the Subprograma Ciência e Tecnologia do $2^{\circ}$ Quadro Comunitário de Apoio is gratefully acknowledged. 


\section{Introduction}

This note presents two simple proofs of a theorem by Harris (1985b) on the existence of subgame perfect equilibria in continuous games of perfect information.

Broadly, both proofs start to solve a simple related problem, which is then extended to the case in which we are interested. In fact, in our first proof we consider a class of games in which it it easy to prove the existence of equilibria; then, we show that any continuous game can be approximated by games in that class and that limit points of equilibrium outcomes of the approximating games is an equilibrium outcome of the original game. Similarly, in our second proof we consider a set of outcomes for which it is easy to show its non-emptiness and then we show that this set is a subset of the set of equilibrium outcomes. In both cases, it follows that the set of equilibrium outcomes is nonempty and a perfect equilibrium can then be constructed from any equilibrium outcome.

In both proofs we start by focusing on games with a finite horizon. Our first proof considers simple games, that is, games in which all players' payoff functions are finitely-valued. For such games it is easy to show that they have a perfect equilibrium, which can be done using the standard backward induction argument. The point is that we can always find an optimal choice for any player given an initial history and the strategies of the others since there is only finitely many values that the payoff function can take.

We then extend this result to continuous games. We first approximate the payoff function of each player by a sequence of simple functions, in a way that is standard in measure and integration theory (see, for example, Wheeden and Zygmund (1977)). This approximation induces a sequence of games with the following property: each game in the sequence differs from the original game only on the payoff function and it has a nonempty set of equilibrium outcomes. We then show that the sequence of the closure of each of those sets shrinks to the set of equilibrium outcomes of the original game, implying that the original game has a nonempty, compact set of equilibrium outcome.

Although inspired by the work of Hellwig, Leininger, Reny, and Robson (1990), this proof differs from theirs in that the only space we discretize is the range of players' payoff functions. This lead us to consider simple games, which are simple enough to prove easily the existence of a perfect equilibrium. Furthermore, it allows us to avoid changing players' action spaces, which 
simplifies the proof of existence of equilibria in general continuous games and dispenses with metric spaces assumptions.

Our second proof relies on the set of nice outcomes introduced by Hellwig and Leininger (1987). It easy to show that the set of such outcomes is nonempty since these outcomes can be characterized as the set of solutions of a maximization problem of a continuous function on a nonempty compact space.

We then use the non-emptiness of the set of nice outcomes to show the existence of an equilibrium outcome. This is done by showing that the set of nice outcome is a subset of the set of equilibrium outcomes. The intuition behind this results is that an equilibrium outcome also solves a maximization problem, but a more restrictive one.

Our second proof is considerably simpler than the one of Hellwig and Leininger (1987). This happens because their use it to obtain a stronger conclusion, namely, the existence of a measurable perfect equilibria. However, we note that, by dropping this goal, not only do we simplify the argument, but also we can dispenses with the assumption that players' action spaces are metric spaces. In fact, both it and our first proof dispense with the assumption that they are Hausdorff spaces used originally by Harris (1985b).

Both proofs rely on a continuity argument on how the equilibrium outcomes of subgames change with the initial history defining it. This argument is done in Lemma 3 below using our approximation by simple games, where we show that accumulation points of equilibrium outcomes of approximating simple games are equilibrium outcomes of the limiting continuous game. A similar argument can be used to show that the correspondence mapping initial histories into equilibrium outcomes of the corresponding subgame is both closed and upper hemicontinuous.

\section{Games of Perfect Information}

Our notation follows closely the one used by Harris (1985b). There is a possibly infinite set of time periods $t=1, \ldots, T \leq \infty$. To each time period, we associate a different player. ${ }^{1}$ For all $1 \leq t \leq T$, player $t$ chooses an action which can be represented by an element of $S_{t}$. An history of the game is an

\footnotetext{
${ }^{1}$ By the single deviation property (see Harris (1985b, Lemma 1)), the assumption that each players moves only once is without loss of generality.
} 
element of

$$
S=\underset{t=0}{X} S_{t},
$$

where $S_{0}=\{0\}$ is included for notational convenience. Given $k \geq 0, x=$ $\left(x_{k}, \ldots, x_{T}\right) \in S_{k} \times \cdots \times S_{T}$, and $t \geq k, \lambda_{t} x=\left(x_{k}, \ldots, x_{t}\right)$ denotes the components of $x$ from $k$ until $t$, and $\mu_{t} x=\left(x_{t}, x_{t+1}, \ldots, x_{T}\right)$ the sequence of outcomes from $t$ onwards.

A game of perfect information is a pair $G=(H, P)$ where $H$ is a nonempty subset of $S, P=\left(P_{1}, \ldots, P_{T}\right)$ and

$$
P_{t}: H \rightarrow \mathbb{R}
$$

for all $1 \leq t \leq T$. The set $H$ denotes the set of all possible histories in the game $G$ and the function $P_{t}$ is player $t$ 's payoff function.

For all $1 \leq t \leq T$ and $x \in H$, let

$$
A_{t}\left(\lambda_{t-1} x\right)=\left\{y_{t}: y \in H, \lambda_{t-1} y=\lambda_{t-1} x\right\}
$$

denote the set of outcomes that are possible in period $t$ given the history $\lambda_{t-1} x$. Note that $A_{t}\left(\lambda_{t-1} x\right)$ defines a correspondence $A_{t}: \lambda_{t-1} H \rightrightarrows H_{t}$ and that $\operatorname{graph}\left(A_{t}\right)=\lambda_{t} H$. In particular, graph $\left(A_{T}\right)=H$.

When player $t$ chooses, it is with perfect information on previous choices by players 1 up to $t-1$ and he can choose any feasible choice. Thus, a strategy for player $t$ is a function

$$
f_{t}: \lambda_{t-1} H \rightarrow S_{t}
$$

such that $f_{t}\left(\lambda_{t-1} x\right) \in A_{t}\left(\lambda_{t-1} x\right)$ for all $x \in H$.

A strategy profile $f$ is a vector of individual strategies: $f=\left(f_{1}, \ldots, f_{T}\right)$. Let $F(H)$ denote the set of all strategy profiles (or strategies, for short), and $F^{t}(H)$ denote the set of all player $t^{\prime}$ 's strategies. If $h^{t} \in F^{t}(H)$, and $f \in F(H)$, then $f \backslash h^{t}$ denotes the strategy resulting from replacing player t's strategy $f^{t}$ by $h^{t}$.

Given $f \in F, x \in H$ and $t \in \mathbb{N}$, the history resulting if $f$ is employed from period $t+1$ onwards after an initial history $\lambda_{t} x$ is denoted by $\alpha[f, x, t] \in H$. A strategy $f$ is a perfect equilibrium of the game $G=(H, P)$ if

$$
P_{t}(\alpha[f, x, t]) \geq P_{t}\left(\alpha\left[f \backslash h^{t}, x, t\right]\right)
$$

for all $1 \leq t \leq T, h^{t} \in F^{t}(H), x \in H$. 
Every initial history of a game with perfect information induces another such games. Formally, if $1 \leq t \leq T$ and $x \in \lambda_{t-1} H$, let $H_{t}(x)=\{y \in H$ : $\left.\lambda_{t-1} y=x\right\}$ be the set of possible histories which can follow the initial history $x$. The subgame starting at $x$ is the game $G_{t}(x)$ with perfect information defined by $\left(H_{t}(x), P_{\mid H_{t}(x)}\right)$.

If $1 \leq t \leq T, x \in \lambda_{t-1} H, f$ is a perfect equilibrium of a game of perfect information $G_{t}(x)$ the history $y \in \mu_{t} H$ defined by $y_{t}=f_{t}(x)$ and $y_{k}=$ $f_{k}\left(x, \lambda_{k-1} y\right)$ for all $k \geq t+1$ is called an equilibrium outcome of $f$. The set of all equilibrium outcomes of $G_{t}(x)$ is denoted by $\Pi_{t}(x) \subseteq \mu_{t} H$.

Let $G=(H, P)$ be a game with perfect information. We say that $G$ is a game with finite horizon if $T$ is finite; otherwise, $G$ is a game with infinite horizon. A game $G$ is simple if $P_{t}$ is simple for all $1 \leq t \leq T$ and is continuous if for all $t=1, \ldots, T$,

1. $S_{t}$ is a compact topological space,

2. $P_{t}$ is continuous,

3. $A_{t}: \lambda_{t-1} \rightrightarrows H_{t}$ is lower hemicontinuous and

4. $H$ is a closed subset of $S$.

\section{Equilibrium Outcomes}

In this section we characterize the set of equilibrium outcomes. We focus on equilibrium outcomes because it is easier to show the non-emptiness of the set of equilibrium outcomes than to establish the existence of an equilibrium strategy directly. The reason is that the set of equilibrium outcomes is compact in all continuous games, while the set of equilibrium strategies typically is not (see Harris (1985a, proposition 4)).

Let $T<\infty$ and for all $x \in \lambda_{T-1} H$, define

$$
E_{T}(x)=\left\{y \in A_{T}(x): P_{T}(x, y) \geq P_{T}(x, z) \text {, for all } z \in A_{T}(x)\right\} .
$$

The set $E_{T}(x)$ gives the equilibrium paths of the one-player game formed by player $T$ with action space $A_{T}(x)$ and payoff function $y \mapsto P_{T}(x, y)$. Assume that $E_{k}(x)$ has been defined for all $k=t+1, \ldots, T$ and $x \in \lambda_{k-1}(x)$. Then, define

$$
\begin{aligned}
E_{t}(x)=\left\{y \in \mu_{t} H:\right. & \mu_{t+1} y \in E_{t+1}\left(x, y_{t}\right) \text { and for all } z \in A_{t}(x) \text { there exists } \\
& \left.w \in E_{t+1}(x, z) \text { such that } P_{t}(x, y) \geq P_{t}(x, z, w)\right\} .
\end{aligned}
$$


Lemma 1 then shows that $E_{t}(x)$ is the set of equilibrium paths of the subgame starting at $x$.

Lemma 1 If $G$ is a game with perfect information with finite horizon, then

$$
\Pi_{t}(x)=E_{t}(x)
$$

for all $t=1, \ldots, T$ and all $x \in \lambda_{t-1} H$.

Proof. We start by showing that $E_{t}(x) \subseteq \Pi_{t}(x)$ for all $t=1, \ldots, T$ and all $x \in \lambda_{t-1} H$.

The proof is by induction. For $k=T$, this is obvious. So, assume that $E_{k}(x) \subseteq \Pi_{k}(x)$ for all $k=t+1, \ldots, T$ and all $x \in \lambda_{k-1} H$. Let $x \in \lambda_{t-1} H$ and $y \in E_{t}(x)$. Then, $\mu_{t+1} y \in E_{t+1}\left(x, y_{t}\right) \subseteq \Pi_{t+1}\left(x, y_{t}\right)$. Thus, there exists a perfect equilibrium $\left(\bar{f}_{t+1}, \ldots, \bar{f}_{T}\right)$ of $G(x)$ satisfying $\bar{f}_{k}\left(x, \lambda_{k-1} y\right)=y_{k}$ for all $k \geq t+1$. Define $f_{t}(x)=y_{t}$ and $f_{k}(s)=\bar{f}_{k}(s)$ for all $s \in H$ such that $\lambda_{t} s=\left(x, y_{t}\right)$ and all $k \geq t+1$.

Let $z \in A_{t}(x)$. Then, there exists $w \in E_{t+1}(x, z) \subseteq \Pi_{t}(x, z)$ such that $P_{t}(x, y) \geq P_{t}(x, z, w)$. Hence, there exists a perfect equilibrium $\left(\tilde{f}_{t+1}, \ldots, \tilde{f}_{T}\right)$ of $G(x)$ satisfying $\tilde{f}_{k}\left(x, z, \lambda_{k-1} w\right)=w_{k}$ for all $k \geq t+1$. Define $f_{k}(s)=\tilde{f}_{k}(s)$ for all $s \in H$ such that $\lambda_{t} s=\left(x, z_{t}\right)$ and all $k \geq t+1$.

By construction, $\left(f_{t}, \ldots, f_{T}\right)$ is a perfect equilibrium of $G(x)$ satisfying $f_{k}\left(x, \lambda_{k-1} y\right)=y_{k}$ for all $k \geq t$. This implies that $y \in \Pi_{t}(x)$.

Finally, we show that $\Pi_{t}(x) \subseteq E_{t}(x)$ for all $t=1, \ldots, T$ and all $x \in \lambda_{t-1} H$, again by induction.

The case $k=T$ is obvious. So, assume that $\Pi_{k}(x) \subseteq E_{k}(x)$ for all $k=$ $t+1, \ldots, T$ and all $x \in \lambda_{k-1} H$. Let $x \in \lambda_{t-1} H$ and $y \in \Pi_{t}(x)$. Then, there exists a perfect equilibrium $\left(f_{t}, \ldots, f_{T}\right)$ of $G(x)$ such that $y_{k}=f_{k}\left(\lambda_{k-1}(x, y)\right)$ for all $k \geq t$.

Since $\mu_{t+1} y \in \Pi_{t+1}\left(x, y_{t}\right)$, then $\mu_{t+1} y \in E_{t+1}\left(x, y_{t}\right)$. Let $z \in A_{t}(x)$ and consider $w$ defined by $w_{t+1}=f_{t+1}(x, z)$ and $w_{k}=f_{k}\left(x, z, \lambda_{k-1} w\right)$ for all $k>t+1$. Then, $w \in \Pi(x, z)$ and so $w \in E_{t+1}(x, z)$. Letting $g_{t}: \lambda_{t-1} H \rightarrow S_{t}$ be defined by $g_{t}(x)=z$ and $g_{t}(s)=f_{t}(s)$ for all $s \neq x$, then $\alpha\left[f \backslash g_{t}, x, t\right]=$ $(x, z, w)$. Since we have that $\alpha[f, x, t]=(x, y)$, it follows that $P_{t}(x, y) \geq$ $P_{t}(x, z, w)$. Hence, $y \in E_{t}(x)$. 


\section{Approximation by Simple Functions and Harris' Theorem}

In this section we show that any simple game with perfect information with finite horizon has a perfect equilibrium. This result is then used to establish the existence of a perfect equilibrium in any continuous game with perfect information with finite horizon.

\subsection{Existence of Perfect Equilibria}

Let $G=(H, P)$ be a game of perfect information with a finite horizon, and assume that for all $t=1, \ldots, T, P_{t}(H) \subset[0,1)$. For all $t=1, \ldots, T$ and $k \in \mathbb{N}$, let $P_{t}^{k}: H \rightarrow \mathbb{R}$ be defined by

$$
P_{t}^{k}(h)=\frac{j-1}{2^{k}} \quad \text { if } \quad \frac{j-1}{2^{k}} \leq P_{t}(h)<\frac{j}{2^{k}},
$$

for $j=1, \ldots, 2^{k}$. Define $G_{k}=\left(H, P^{k}\right)$, for all $k \in \mathbb{N}$. The sequence $\left\{P^{k}\right\}$ has the following important properties: for all $t=1, \ldots, T$ and all $k \in \mathbb{N}$,

1. $\left\|P_{t}^{k}-P_{t}\right\|_{\infty} \leq \frac{1}{2^{k}}$

2. For all $h, l \in H, P_{t}(h) \geq P_{t}(l)$ implies $P_{t}^{k}(h) \geq P_{t}^{k}(l)$;

3. For all $h, l \in H, P_{t}^{k+1}(h) \geq P_{t}^{k+1}(l)$ implies $P_{t}^{k}(h) \geq P_{t}^{k}(l)$; and

4. $P_{t}^{k}$ is a simple function.

This last property allows us to solve the game $G_{k}$ by backwards induction, and thus show that it has a perfect equilibrium. More generaly, this is a consequence of the fact that every simple game with finite horizon has a perfect equilibrium.

Lemma 2 Every simple game with perfect information with finite horizon has a perfect equilibrium.

Proof. Let $G$ be a simple game with perfect information with finite horizon. We will prove the lemma by backward induction.

Let $k \in \mathbb{N}$. Let $x \in \lambda_{T-1} H$. Since $P_{T}$ is simple, then $\left\{P_{T}(x, y): y \in\right.$ $\left.A_{T}(x)\right\}$ is finite, and so there exists $y^{*} \in A_{T}(x)$ that maximizes $y \mapsto P_{T}(x, y)$ 
in $A_{T}(x)$. Thus, define $f_{T}(x)=y^{*}$. This defines an optimal strategy $f_{T}$ for player $T$.

Suppose that we have defined $f_{n}: \lambda_{n-1} H \rightarrow S_{n}$ such that $f_{n}(x) \in A_{n}(x)$ and

$$
P_{n}\left(\alpha\left[\left(f_{n+1}, \ldots, f_{T}\right),\left(x, f_{n}(x)\right), n+1\right]\right) \geq P_{n}\left(\alpha\left[\left(f_{n+1}, \ldots, f_{T}\right),(x, z), n+1\right]\right)
$$

for all $n=t+1, \ldots, T, x \in \lambda_{n-1} H$ and $z \in A_{n}(x)$.

Let $x \in \lambda_{t-1} H$. Since $P_{t}$ is simple, then the set

$$
\left\{P_{t}\left(\alpha\left[\left(f_{t+1}, \ldots, f_{T}\right),(x, z), t+1\right]\right): z \in A_{t}(x)\right\}
$$

is finite, and so there exists $z^{*}$ that maximizes

$$
z \mapsto P_{t}\left(\alpha\left[\left(f_{t+1}, \ldots, f_{T}\right),(x, z), t+1\right]\right)
$$

in $A_{t}(x)$. Thus, define $f_{t}(x)=z^{*}$. This defines an optimal strategy $f_{t}$ for player $t$.

Let $f=\left(f_{1}, \ldots, f_{t}\right)$. Let $1 \leq t \leq T, x \in \lambda_{t-1} H$ and $g_{t} \in F^{t}$. Since $\alpha[f, x, t]=\alpha\left[\left(f_{t+1}, \ldots, f_{T}\right),\left(x, f_{t}(x)\right), t+1\right]$ and

$$
\alpha\left[f \backslash g_{t}, x, t\right]=\alpha\left[\left(f_{t+1}, \ldots, f_{T}\right),\left(x, g_{t}(x)\right), t+1\right],
$$

it follows that $P_{t}(\alpha[f, x, t]) \geq P_{t}\left(\alpha\left[f \backslash g_{t}, x, t\right]\right)$. Thus, $f=\left(f_{1}, \ldots, f_{T}\right)$ is a perfect equilibrium.

We will prove Harris' theorem by approximating a given game $G$ by a sequence of games $\left\{G_{k}\right\}_{k=1}^{\infty}$ as defined above. In fact, this will be a consequence of Theorem 1 below, which shows that the set of equilibrium outcomes of $G_{k}$ converges to the set of equilibrium outcomes of $G$.

Theorem 1 If $G=(H, P)$ is a continuous game of perfect information with finite horizon, then

$$
E_{t}(x)=\bigcap_{k=1}^{\infty} E_{t}^{k}(x)=\bigcap_{k=1}^{\infty} \overline{E_{t}^{k}(x)}
$$

for all $t=1, \ldots, T$ and $x \in \lambda_{t-1} H$.

Proof. First note that because $H$ is compact and $P_{t}$ is continuous for all $t$, there is no loss in generality by assuming that $P_{t}(H) \subset[0,1)$ for all $t$. For $k \in \mathbb{N}$, let $G_{k}$ be as defined above. 
We show first that $E_{t}(x) \subseteq \cap_{k=1}^{\infty} E_{t}^{k}(x)$ for all $1 \leq t \leq T$ and $x \in \lambda_{t-1} H$. This follows from the second property of the sequence $\left\{P^{k}\right\}$. For player $T$, this is obvious: if $x \in \lambda_{T-1} H$ and $y \in E_{T}(x)$, then $P_{T}(x, y) \geq P_{T}(x, z)$ for all $z \in A_{T}(x)$ and so $P_{T}^{k}(x, y) \geq P_{T}^{k}(x, z)$ for all $k \in \mathbb{N}$. Thus, $y \in E_{T}^{k}(x)$ for all $k \in \mathbb{N}$.

So, suppose that $E_{n}(x) \subseteq \cap_{k=1}^{\infty} E_{n}^{k}(x)$ for all $n \geq t+1$ and $x \in \lambda_{n-1} H$. Let $x \in \lambda_{t-1} H, y \in E_{t}(x)$ and $k \in \mathbb{N}$. Then, $\mu_{t+1} y \in E_{t+1}\left(x, y_{t}\right) \subseteq E_{t+1}^{k}\left(x, y_{t}\right)$. Let $z \in A_{t}(x)$. Then, there exists $w \in E_{t+1}(x, z) \subseteq E_{t+1}^{k}(x, z)$ such that $P_{t}(x, y) \geq P_{t}(x, z, w)$. Hence, $P_{t}^{k}(x, y) \geq P_{t}^{k}(x, z, w)$ and so $y \in E_{t}^{k}(x)$.

We show next that $\cap_{k=1}^{\infty} \overline{E_{t}^{k}(x)} \subseteq E_{t}(x)$ for all $t=1, \ldots, T$ and $x \in \lambda_{t-1} H$. Let $1 \leq t \leq T, x \in \lambda_{t-1} H$ and $y \in \cap_{k=1}^{\infty} \overline{E_{t}^{k}(x)}$. Then, for each $k \in \mathbb{N}$, there a net $\left\{y_{j}^{k}\right\}_{j \in J_{k}} \subseteq E_{t}^{k}(x)$ converging to $y$. Letting $y^{k}=y$ and $x_{j}^{k}=x^{k}=x$ for all $k \in \mathbb{N}$ and $j \in J_{k}$, it follows from Lemma 3 that $y \in E_{t}(x)$.

The following lemma is used in Theorem 1 and constitutes the crucial argument in its proof.

Lemma 3 Let $G$ be a continuous game with perfect information with finite horizon.

Let $1 \leq t \leq T, x \in \lambda_{t-1} H$ and $y \in \lambda_{t} H$. Let $\left\{x^{k}\right\}_{k \in K}$ and $\left\{y^{k}\right\}_{k \in K}$ be nets converging to $x$ and $y$, respectively. Let $N: K \rightarrow \mathbb{N}$ be such that $N(k) \rightarrow \infty$. For all $k \in K$, let $\left\{x_{j}^{k}\right\}_{j \in J_{k}}$ and $\left\{y_{j}^{k}\right\}_{j \in J_{k}}$ be nets such that $x_{j}^{k} \rightarrow_{j} x^{k}, y_{j}^{k} \rightarrow_{j} y^{k}$ and $y_{j}^{k} \in E_{t}^{N(k)}\left(x_{j}^{k}\right)$ for all $j \in J_{k}$. Then, $y \in E_{t}(x)$.

Proof. The proof is by (backward) induction. In the case of player $T$, note first that $y^{k} \in A_{T}\left(x^{k}\right)$ for all $k \in K$ and so $y \in A_{T}(x)$. This follows since $H$ is closed and $\operatorname{graph}\left(A_{T}\right)=H$. So, it remains to show that $P_{T}(x, y) \geq P_{T}(x, z)$ for all $z \in A_{T}(x)$.

Let $z \in A_{T}(x)$. By Lemma 5 , there exists a subnet $\left\{\tilde{x}^{m}\right\}_{m \in M}$ of $\left\{x^{k}\right\}_{k \in K}$ and a corresponding net $\left\{z^{m}\right\}_{m \in M}$ such that $z^{m} \in A_{T}\left(\tilde{x}^{m}\right)$ for all $m \in M$ and $z^{m} \rightarrow z$. Let $\alpha: M \rightarrow K$ be the admissible function defining the subnet, i.e., $\tilde{x}_{m}=x_{\alpha(m)}$ for all $m \in M$. Let $\left\{\tilde{y}^{m}\right\}_{m \in M}$ be the corresponding subnet of $\left\{y^{k}\right\}_{k \in K}$.

Let $m \in M$ and $\tilde{x}_{j}^{m}=x_{j}^{\alpha(m)}$ for all $j \in \tilde{J}_{m}=J_{\alpha(m)}$. Then, $\tilde{x}_{j}^{m} \rightarrow \tilde{x}^{m}$. By Lemma 5, there exist a subnet $\left\{\bar{x}_{l}^{m}\right\}_{l \in L_{m}}$ of $\left\{\tilde{x}_{j}^{m}\right\}_{j \in \tilde{J}_{m}}$ and a corresponding net $\left\{z_{l}^{m}\right\}_{l \in L_{m}}$ such that $z_{l}^{m} \in A_{T}\left(\bar{x}_{l}^{m}\right)$ for all $l \in L_{m}$ and $z_{l}^{m} \rightarrow z^{m}$. Let $\left\{\bar{y}_{l}^{m}\right\}_{l \in L_{m}}$ be the corresponding subnet of $\left\{\tilde{y}^{m}\right\}_{m \in M}$. 
Let $m \in M$ and $l \in L_{m}$. Since $\bar{y}_{l}^{m} \in E_{t}^{N(\alpha(m))}\left(\bar{x}_{l}^{m}\right)$, then $P_{T}^{N(\alpha(m))}\left(\bar{x}_{l}^{m}, \bar{y}_{l}^{m}\right) \geq$ $P_{T}^{N(\alpha(m))}\left(\bar{x}_{l}^{m}, z_{l}^{m}\right)$ and so

$$
P_{T}\left(\bar{x}_{l}^{m}, \bar{y}_{l}^{m}\right) \geq P_{T}\left(\bar{x}_{l}^{m}, z_{l}^{m}\right)-\frac{2}{2^{N(\alpha(m))}} .
$$

Hence, $P_{T}\left(\tilde{x}^{m}, \tilde{y}^{m}\right) \geq P_{T}\left(\tilde{x}^{m}, z^{m}\right)-2 / 2^{N(\alpha(m))}$ and so, we obtain $P_{T}(x, y) \geq$ $P_{T}(x, z) .^{2}$ Thus, $y \in E_{T}(x)$.

Suppose that the claim is true for all $n \geq t+1$. Let $x \in \lambda_{t-1} H, y \in \lambda_{t} H$ and $\left\{x_{j}^{k}\right\}_{j},\left\{y_{j}^{k}\right\}_{j},\left\{x^{k}\right\}_{k}$ and $\left\{y^{k}\right\}_{k}$ be as in the statement of the claim. Since $\left(x^{k}, y_{t}^{k}\right) \rightarrow\left(x, y_{t}\right), \mu_{t+1} y^{k} \rightarrow \mu_{t+1} y$ and for all $k, \mu_{t+1} y_{j}^{k} \in E_{t+1}^{N(k)}\left(x_{j}^{k}, y_{j t}^{k}\right)$ for all $j,\left(x_{j}^{k}, y_{j t}^{k}\right) \rightarrow\left(x^{k}, y_{t}^{k}\right)$, and $\mu_{t+1} y_{j}^{k} \rightarrow \mu_{t+1} y^{k}$, it follows that $\mu_{t+1} y \in$ $E_{t+1}\left(x, y_{t}\right)$.

Let $z \in A_{t}(x)$. By Lemma 5 , there exists a subnet $\left\{\tilde{x}^{m}\right\}_{m \in M}$ of $\left\{x^{k}\right\}_{k \in K}$ and a corresponding net $\left\{z^{m}\right\}_{m \in M}$ such that $z^{m} \in A_{T}\left(\tilde{x}^{m}\right)$ for all $m \in M$ and $z^{m} \rightarrow z$. Let $\alpha: M \rightarrow K$ be the admissible function defining the subnet, i.e., $\tilde{x}_{m}=x_{\alpha(m)}$ for all $m \in M$. Let $\left\{\tilde{y}^{m}\right\}_{m \in M}$ be the corresponding subnet of $\left\{y^{k}\right\}_{k \in K}$.

Let $m \in M$ and $\tilde{x}_{j}^{m}=x_{j}^{\alpha(m)}$ for all $j \in \tilde{J}_{m}=J_{\alpha(m)}$. Then, $\tilde{x}_{j}^{m} \rightarrow \tilde{x}^{m}$. By Lemma 5 , there exist a subnet $\left\{\bar{x}_{l}^{m}\right\}_{l \in L_{m}}$ of $\left\{\tilde{x}_{j}^{m}\right\}_{j \in \tilde{J}_{m}}$ and a corresponding net $\left\{z_{l}^{m}\right\}_{l \in L_{m}}$ such that $z_{l}^{m} \in A_{T}\left(\bar{x}_{l}^{m}\right)$ for all $l \in L_{m}$ and $z_{l}^{m} \rightarrow z^{m}$. Let $\left\{\bar{y}_{l}^{m}\right\}_{l \in L_{m}}$ be the corresponding subnet of $\left\{\tilde{y}^{m}\right\}_{m \in M}$.

Let $m \in M$ and $l \in L_{m}$. Since $\bar{y}_{l}^{m} \in E_{t}^{N(\alpha(m))}\left(\bar{x}_{l}^{m}\right)$, then there exists $w_{l}^{m} \in E_{t+1}^{N(\alpha(m))}\left(\bar{x}_{l}^{m}, z_{l}^{m}\right)$ such that $P_{T}^{N(\alpha(m))}\left(\bar{x}_{l}^{m}, \bar{y}_{l}^{m}\right) \geq P_{T}^{N(\alpha(m))}\left(\bar{x}_{l}^{m}, z_{l}^{m}, w_{l}^{m}\right)$. Since $\mu_{t+1} H$ is compact, we may assume that $\left\{w_{l}^{m}\right\}_{l \in L_{m}}$ converges; let $w^{m}$ denote one of its limit points. Again, taking a subnet if necessary, we may assume that $\left\{w^{m}\right\}_{m \in M}$ converges and let $w$ be one of its limit points.

Since $w_{l}^{m} \in E_{t+1}^{N(\alpha(m))}\left(\bar{x}_{l}^{m}, z_{l}^{m}\right)$ for all $m \in M$ and $l \in L_{m}, \bar{x}_{l}^{m} \rightarrow \tilde{x}^{m}$, $z_{l}^{m} \rightarrow z^{m}, \tilde{x}^{m} \rightarrow x, z^{m} \rightarrow z$ and $N(\alpha(m)) \rightarrow \infty$, then $w \in E_{t+1}(x)$. Furthermore, we have that

$$
P_{T}\left(\bar{x}_{l}^{m}, \bar{y}_{l}^{m}\right) \geq P_{T}\left(\bar{x}_{l}^{m}, z_{l}^{m}, w_{l}^{m}\right)-\frac{2}{2^{N(\alpha(m))}},
$$

which implies that $P_{T}(x, y) \geq P_{T}(x, z, w)$. Thus, $y \in E_{t}(x)$.

\footnotetext{
${ }^{2}$ Note that $2 / 2^{N(\alpha(m))}$ converges to zero since $N(\alpha(m))$ converges to infinity. The latter assertion can be shown as follows: For all $n \in \mathbb{N}$, there exists $k_{0} \in K$ such that $k \succeq k_{0}$ implies that $N(k) \geq n$. Also, there is $m_{0} \in M$ such that $m \succeq m_{0}$ implies that $\alpha(m) \succeq k_{0}$. Hence, for all $m \succeq m_{0}$, it follows that $N(\alpha(m)) \geq n$.
} 
Theorem 1 gives a characterization of subgame perfect equilibrium paths of $G$ in terms of the equilibrium paths of the approximating games, and provides an algorithm for their computation. Also, it follows immediately from Theorem 1 that $E_{t}(x)$ is compact and closed for all $1 \leq t \leq T$ and $x \in \lambda_{t-1} H$.

We can derive further properties of the set of equilibrium outcomes using Lemma 3. Note that the set $E_{t}(x)$, with $1 \leq t \leq T$ and $x \in \lambda_{t-1} H$, defines a correspondence $E_{t}: \lambda_{t-1} H \rightrightarrows \mu_{t} H$. This correspondence assigns to every initial history the set of equilibrium outcomes of the corresponding outcomes. A simple modification of Lemma 3 (replace $E_{t}^{N(k)}\left(x_{j}^{k}\right)$ by $E_{t}\left(x_{j}^{k}\right)$ in the its statement) together with Theorem 7.1.16 of Klein and Thompson (1984) then imply the following properties for $E_{t}$.

Theorem 2 If $G$ is a continuous game with perfect information with finite horizon, then the correspondence $E_{t}$ is closed-valued, closed and upper hemicontinuous for all $1 \leq t \leq T$.

Another important question is whether or not $E_{t}$ is nonempty-valued, which is answered affirmatively by Harris' Theorem. As we show below, it follows easily from Lemma 2 and Theorem 1.

Theorem 3 (Harris) Every continuous game with perfect information with finite horizon has a perfect equilibrium.

Proof. Let $G$ be a continuous game with perfect information with finite horizon. For all $k \in \mathbb{N}$, let $G_{k}$ be as defined above. By Lemma 2, it follows that $E_{1}^{k}$ is nonempty. Since, for all $k \in \mathbb{N}, 1 \leq t \leq T$ and $h, l \in H$, $P_{t}^{k+1}(h) \geq P_{t}^{k+1}(l)$ implies $P_{t}^{k}(h) \geq P_{t}^{k}(l)$, it follows that $E_{t}^{k+1}(x) \subseteq E_{t}^{k}(x)$ for all $x \in \lambda_{t-1} H$.

Note that $S_{1} \times \cdots \times S_{T}$ is compact. Since $\overline{E_{1}^{k}}$ is a nonempty, closed subset of $S_{1} \times \cdots \times S_{T}$ for all $k \in \mathbb{N}$, it follows that $\cap_{k=1}^{\infty} \overline{E_{1}^{k}}$ is a nonempty subset of $S_{1} \times \cdots \times S_{T}$ (see Kelley (1955, theorem 1, page 136)). Hence, it follows from Theorem 1 that $E_{1}$ is nonempty.

Remark 1 Note that the above Theorem dispenses with the assumption used by Harris (1985b) that $S_{t}$ is Hausdorff for all $1 \leq t \leq T$. 


\subsection{Relation with Hellwig et al. (1990)}

Our approach in this section is similar to that of Hellwig, Leininger, Reny, and Robson (1990). In fact, both have the same structure:

1. Find a class of finite horizon games of perfect information in which it easy to prove the existence of a perfect equilibrium;

2. Approximate general games with a finite horizon with games in that class;

3. Show that the limit of equilibrium outcomes of the approximating games is an equilibrium outcome of the original game;

4. Construct a perfect equilibrium from the equilibrium outcome; and

5. Extend the existence result for games with an infinite horizon.

Despite using the same structure, our proof differs from theirs. It starts by differing on the class of easy games we consider: games with finite-valued (i.e., simple) payoff functions in our case and finite-action games in theirs. Note, however, that all payoff functions are finite-valued in a finite-action game and that this is enough to show the existence of perfect equilibrium easily by backward induction. This shows that the ability to prove existence of equilibria easily does not really depend on whether the action space is finite or not but rather on whether players' payoff functions are finite-valued or not.

Step 3 is the crucial step in the proof of existence of perfect equilibria in general finite horizon games. How easy this argument is depends on how good are the approximations we consider. The approximation we use is particularly strong, not only because payoff functions converge uniformly, but also because the ordering of outcomes is refined along the sequence of the approximations. This makes the proof of Theorems 1 and 2 simple. Furthermore, the proof of Lemma 3, which is similar to part of Theorem 1 of Hellwig, Leininger, Reny, and Robson (1990), is also simplified since, in contrast to them, all approximating games have the same action space.

We note that both approaches can be further related by constructing an approximating sequence of simple games from an approximating sequence of finite-action games. Let $G$ be a finite horizon game of perfect information. Assume that $S_{t}$ is a compact metric space for all $1 \leq t \leq T$ and let $d_{t}$ denote 
the metric on $S_{t}$. For all $k \in \mathbb{N}$, let $S_{t}^{k}$ be a finite subset of $S_{t}$ and assume that $\varepsilon_{t}^{k}=\max _{x \in S_{t}} \min _{y \in S_{t}^{k}} d_{t}(x, y) \rightarrow 0$. Then, the family $\left\{B_{\varepsilon_{t}^{k}}(y)\right\}_{y \in S_{t}^{k}}$ of open balls with radius $\varepsilon_{t}^{k}$ around all points of $S_{t}^{k}$ covers of $S_{t}$ and can be transformed into a disjoint collection $\left\{B_{t}^{k}(y)\right\}_{y \in S_{t}^{k}}$ as follows: order $S_{t}^{k}$, and let $B_{t}^{k}\left(y_{1}\right)=B_{\varepsilon_{t}^{k}}\left(y_{1}\right)$ and $B_{t}^{k}\left(y_{j}\right)=B_{\varepsilon_{t}^{k}}\left(y_{j}\right) \backslash\left(\cup_{l=1}^{j-1} B_{\varepsilon_{t}^{k}}\left(x_{l}\right)\right)$, for all $j \geq 2$. Then, for all $x=\left(x_{0}, x_{1}, \ldots, x_{t}\right) \in H$ define $P_{t}^{k}(x)=P_{t}(s)$ if $x_{t} \in B_{t}^{k}\left(y_{t}\right)$ for all $1 \leq t \leq T$. It is clear that $\left\{P_{t}^{k}\right\}_{k}$ is a sequence of simple functions that converges uniformly to $P_{t}$ for all $t$. However, in general, it does not satisfy the additional property that the ordering of outcomes is refined along the sequence, a property that simplifies the proof.

Despite being more general, using simple games rather than discrete games allows us to address the existence question without having to use metric space techniques. We note, however, that our proof also uses a discretization argument on a metric space, but this is done on the range of players' payoff functions, which is a subset of the real numbers. Since this is the only space we need to discretize (in particular, we do not have to change players' action spaces), we can dispense with the metric space assumptions they make.

\section{$5 \quad$ Nice Outcomes and Harris' Theorem}

An alternative way to establish the existence of an equilibrium outcome is through the use of nice outcomes, introduced by Hellwig and Leininger (1987). This is so, because as we show below, the set of nice outcomes is a subset of the set of equilibrium outcomes. Since one easily shows that the set of nice outcomes is nonempty, it follows that a perfect equilibrium exists.

\section{$5.1 \quad$ Nice Equilibrium Outcomes}

Let $G$ be a continuous game with perfect information with finite horizon. For all $x \in \lambda_{T-1} H$, define $\Phi_{T}(x)=E_{T}(x)$ and $\bar{\Phi}_{T}(x)=\left\{y \in A_{T}(x)\right.$ : $\left.(x, y) \in \overline{\operatorname{graph}\left(\Phi_{T}\right)}\right\}$. By remark 2 , it follows that $E_{T}(x)=\bar{\Phi}_{T}(x)$ and that the correspondence $\bar{\Phi}_{T}: \lambda_{T-1} H \rightrightarrows \mu_{T} H$ is closed. Assume that $\bar{\Phi}_{k}(x)$ has been defined and that the correspondence $\bar{\Phi}_{k}: \lambda_{k-1} H \rightrightarrows \mu_{k} H$ is closed for 
all $k=t+1, \ldots, T$ and $x \in \lambda_{k-1}(x)$. Then, define

$$
\begin{aligned}
\Phi_{t}(x)=\left\{y \in \mu_{t} H:\right. & \mu_{t+1} y \in \bar{\Phi}_{t+1}\left(x, y_{t}\right) \text { and } P_{t}(x, y) \geq P_{t}(x, z, w) \\
& \text { for all } \left.z \in A_{t}(x) \text { and } w \in \bar{\Phi}_{t+1}(x, z)\right\}
\end{aligned}
$$

and $\bar{\Phi}_{t}(x)=\left\{y \in \mu_{t} H:(x, y) \in \overline{\operatorname{graph}\left(\Phi_{t}\right)}\right\}$. The correspondence $\bar{\Phi}_{t}$ has the following important properties.

Theorem 4 If $G$ is a continuous game with perfect information with finite horizon, then $\bar{\Phi}_{t}(x)$ is nonempty and $\bar{\Phi}_{t}(x) \subseteq E_{t}(x)$ for all $1 \leq t \leq T$ and $x \in \lambda_{t-1} H$,

Proof. Let $1 \leq t \leq T$ and $x \in \lambda_{t-1} H$. Define a correspondence $\Lambda_{t}$ : $H_{t} \rightrightarrows \mu_{t+1} H$ by $\Lambda_{t}\left(y_{t}\right)=\bar{\Phi}_{t+1}\left(x, y_{t}\right)$ and let $C_{t}=\operatorname{graph}\left(\Lambda_{t}\right)$. Then, $C_{t}$ is a closed, and hence compact, subset of $\chi_{k=t}^{T} S_{k}$. Since $\Phi_{t}(x)$ equals the set of solutions to the problem

$$
\max _{y \in C_{t}} P_{t}(x, y),
$$

it follows by Weierstrass' Theorem (see Kelley (1955, Exercise 5.A.(b))) that $\Phi_{t}(x)$ is nonempty. Hence, $\bar{\Phi}_{t}(x)$ is nonempty.

We prove that $\bar{\Phi}_{t}(x) \subseteq E_{t}(x)$ by (backward) induction. We have that $E_{T}(x)=\bar{\Phi}_{T}(x)$ for all $x \in \lambda_{T-1} H$. Hence, assume that $\bar{\Phi}_{k}(x) \subseteq E_{k}(x)$ for all $k=t+1, \ldots, T$ and $x \in \lambda_{k-1} H$.

Let $x \in \lambda_{t-1} H$ and $y \in \Phi_{t}(x)$. Then, $\mu_{t+1} y \in \bar{\Phi}_{t+1}\left(x, y_{t}\right) \subseteq E_{t+1}\left(x, y_{t}\right)$. Let $z \in A_{t}(x)$ and let $w \in \bar{\Phi}_{t+1}(x, z)$. Then, $P_{t}(x, y) \geq P_{t}(x, z, w)$ since $y \in \Phi_{t}(x)$. Thus, $y \in E_{t}(x)$.

This implies that $\operatorname{graph}\left(\Phi_{t}\right) \subseteq \operatorname{graph}\left(E_{t}\right)$. Since $\operatorname{graph}\left(E_{t}\right)$ is closed, it follows that $\overline{\Phi_{t}(x)} \subseteq E_{t}(x)$.

Clearly, Theorem 4 shows that $E_{1}$ is nonempty and so $G$ has a perfect equilibrium. Thus, we obtain an alternative proof Harris' Theorem. Again, we emphasized that this proof also shows that we can dispense with the assumption that $S_{t}$ is Hausdorff for all $1 \leq t \leq T$.

\subsection{Relation with Hellwig and Leininger (1987)}

Our approach in this section is clearly related with that of Hellwig and Leininger (1987). The proof relies on finding a special subset of the equilibrium outcomes, whose non-emptiness is easy to establish. One such set is the set of of nice outcomes introduced by Hellwig and Leininger (1987). 
However, since the set of nice outcomes is typically a proper subset of the set of equilibrium outcomes, they do not provide a characterization of these. Hence, nice outcomes cannot be used to study the continuity properties of the correspondence mapping initial histories into equilibrium outcomes of the corresponding subgame.

We note that Hellwig and Leininger (1987) have introduced such set in order to show the existence measurable perfect equilibria, a question that we do not address here. Since we care only about existence of equilibria and not about its regularity properties, we obtain a considerably simpler proof. Furthermore, our proof dispenses with metric spaces techniques used by them, and in fact, dispenses with the assumption that the outcome space is Hausdorff.

\section{Games with an Infinite Horizon}

In this section, we extent our result to the case of a countable number of players using a "truncation argument" similar to the one used by Harris (1985b, section 4.4, page 624). ${ }^{3}$ Due to this, we shall be brief.

Let $G$ be a continuous game with perfect information with an infinite horizon. For all $n, t \in \mathbb{N}$ and $x \in \lambda_{t-1}(x)$, let $E_{t}^{n}(x)=\mu_{t} H$ if $t>n$ and

$$
\begin{aligned}
E_{t}^{n}(x)=\left\{y \in \mu_{t} H:\right. & \mu_{t+1} y \in E_{t+1}^{n}\left(x, y_{t}\right) \text { and for all } z \in A_{t}(x) \text { there exists } \\
& \left.w \in E_{t+1}^{n}(x, z) \text { such that } P_{t}(x, y) \geq P_{t}(x, z, w)\right\}
\end{aligned}
$$

if $t \leq n$.

An argument similar to the one used in Section 4 shows that $E_{t}^{n}(x)$ is nonempty and closed for all $n, t \in \mathbb{N}$ and $x \in \lambda_{t-1} H$. Furthermore, an easy inductive argument establishes that $E_{t}^{n+1}(x) \subseteq E_{t}^{n}(x)$ for all $n, t \in \mathbb{N}$ and $x \in \lambda_{t-1} H$. Hence, $E_{t}(x)=\cap_{n=1}^{\infty} E_{t}^{n}(x)$ is nonempty, closed and compact for all $t \in \mathbb{N}$ and $x \in \lambda_{t-1} H$. Furthermore, $E_{t}(x)$ can be described as in Section 3 .

Lemma 4 If $G$ is a continuous game with perfect information with an infinite horizon, then

$$
\begin{aligned}
E_{t}(x)=\left\{y \in \mu_{t} H:\right. & \mu_{t+1} y \in E_{t+1}\left(x, y_{t}\right) \text { and for all } z \in A_{t}(x) \text { there exists } \\
& \left.w \in E_{t+1}(x, z) \text { such that } P_{t}(x, y) \geq P_{t}(x, z, w)\right\}
\end{aligned}
$$

\footnotetext{
${ }^{3}$ See also Börgers (1989) and Börgers (1991) for a related approach.
} 
for all $t \in \mathbb{N}$ and $x \in \lambda_{t-1} H$.

Proof. Let $t \in \mathbb{N}$ and $x \in \lambda_{t-1} H$. Let $y \in E_{t}(x)$. Then, $y \in \cap_{n} E_{t}^{n}(x)$. Hence, $\mu_{t+1} y \in E_{t+1}^{n}(x)$ for all $n$, and so $\mu_{t+1} y \in E_{t+1}(x)$.

Let $z \in A_{t}(x)$. Then, for all $n$, there exists $w_{n} \in E_{t+1}^{n}(x)$ such that $P_{t}(x, y) \geq P_{t}\left(x, z, w_{n}\right)$. Since $\left\{w_{n}\right\}_{n} \subseteq \chi_{k=t+1}^{\infty} S_{k}$ and $\chi_{k=t+1}^{\infty} S_{k}$ is compact, then there exists a converging subnet $\left\{\tilde{w}_{j}\right\}_{j \in J}$ of $\left\{w_{n}\right\}_{n}$. Let $w$ be such that $\tilde{w}_{j} \rightarrow w$. Let $n \in \mathbb{N}$ and $j_{0} \in J$ be such that $j \succeq j_{0}$ implies $N(j) \geq n$. Then, for all $j \succeq j_{0}, \tilde{w}_{j}=w_{N(j)} \in E_{t+1}^{N(j)}(x) \subseteq E_{t+1}^{n}(x)$. Hence, $w \in E_{t+1}^{n}(x)$. Since $n$ is arbitrary, it follows that $w \in E_{t+1}(x)$. Furthermore, $P_{t}(x, y) \geq P_{t}\left(x, z, \tilde{w}_{j}\right)$ for all $j \in J$ implies $P_{t}(x, y) \geq P_{t}(x, z, w)$. Hence, $y \in E_{t}(x)$.

The existence of a perfect equilibrium follows from the non-emptiness of $E_{1}$ in the standard way (see Harris (1985b), Hellwig and Leininger (1987) and Hellwig, Leininger, Reny, and Robson (1990)). If $y \in E_{1}$, define $f_{1}=y_{1}$ and $f_{t}\left(\lambda_{t-1} y\right)=y_{t}$ for all $t>1$. This already defines $f_{1}$. To define $f_{2}$, let $x \in \lambda_{1} H$. If $x=y_{1}$, we already have defined $f_{2}(x)=f_{2}\left(y_{1}\right)=y_{2}$; otherwise, let $w=\left(w_{2}, w_{3}, \ldots\right) \in E_{2}(x)$ and define $f_{2}(x)=w_{2}$ and $f_{t}\left(x, \lambda_{t-1} w\right)=w_{t}$ for all $t>2$. Proceeding in this way, we define a strategy $f$, which is clearly a perfect equilibrium.

\section{Concluding Remarks}

We presented two simple proofs of the existence of a perfect equilibrium in games with perfect information. Both share some important elements with others available. Namely, we focus on the non-emptiness of the set of equilibrium outcomes in games with a finite horizon. This basic result is then used to construct a perfect equilibrium strategy and, furthermore, it is extended to the case of an infinite horizon.

While our argument to extend to the case of an infinite horizon is standard, our construction of a perfect equilibrium from an equilibrium outcomes in finite horizon games is not. Typically, this is done by defining the equilibrium strategies using forward induction. Although this must be the case with an infinite horizon (since there is no terminal period), it can be done by backward induction when the horizon is finite and in a similar way as it is standard in finite-action, finite horizon games with perfect information.

Despite this more transparent construction, our main contributions regard the non-emptiness of the set of equilibrium outcomes. We first establish 
it using an approximation by simple games. Although inspired by the work of Hellwig, Leininger, Reny, and Robson (1990), it differs from theirs in that the only space we discretize is the range of players' payoff functions. This lead us to consider simple games, which are simple enough to prove easily the existence of a perfect equilibrium. Furthermore, it allows us to avoid changing players' action spaces, which simplifies the proof of existence of equilibria in general continuous games and dispenses with metric spaces assumptions.

Our second proof relies on the set of nice outcomes introduced by Hellwig and Leininger (1987). The advantage of focusing on nice outcomes is that these outcomes can be characterized as the set of solutions of a maximization problem of a continuous function on a nonempty compact space. This implies that the set of nice outcomes is nonempty. Since we can further show that the set nice outcome is a subset of the set of equilibrium outcomes, we obtain the existence of equilibria.

Both proofs rely on a continuity argument on how the equilibrium outcomes of subgames change with the initial history defining it. This argument is done in Lemma 3 below using our approximation by simple games, where we show that accumulation points of equilibrium outcomes of approximating simple games are equilibrium outcomes of the limiting continuous game. A similar argument can be used to show that the correspondence mapping initial histories into equilibrium outcomes of the corresponding subgame is both closed and upper hemicontinuous.

\section{A Appendix}

In this section we provide the definition of some of the mathematical concepts we have used. Furthermore, we present a result on lower hemicontinuous correspondences for which we were unable to find a reference.

The following are standard definitions (see Kelley (1955)). A binary relation $\succeq$ directs a set $D$ if $D$ is nonempty, $\succeq$ is transitive, reflexive and satisfies the following property: if $m, n \in D$, then there is $p \in D$ such that $p \succeq m$ and $p \succeq n$. A net is a pair $(S, \succeq)$ such that $S$ is a function and $\succeq$ directs the domain of $S$. It is typically written as $\left\{S_{n}, n \in D, \succeq\right\}$ where $D$ is the domain of $S$, or as $\left\{S_{n}\right\}_{n \in D}$, leaving the binary relation $\succeq$ implicit.

A net $\left\{S_{n}\right\}_{n \in D}$ is eventually in a set $A$ if there is $m \in D$ such that $S_{n} \in A$ for all $n \succeq m$. A net in a topological space $(X, \tau)$ converges to $x$ relative to $\tau$ if the net is eventually in each $\tau$ - neighborhood of $x$. 
Given two directed sets $E$ and $D$, a function $N: E \rightarrow D$ is admissible if for all $m \in D$ there is $n \in E$ with the property that $p \succeq n$ implies $N(p) \succeq m$. A net $\left\{T_{m}\right\}_{m \in E}$ is a subnet of a net $\left\{S_{n}\right\}_{n \in D}$ if there exists an admissible function $N: E \rightarrow D$ such that $T_{m}=S_{N(m)}$ for all $m \in E$. Clearly, if $S_{n} \rightarrow s$ and $\left\{T_{m}\right\}_{m \in E}$ is a subnet of $\left\{S_{n}\right\}_{n \in D}$, then $T_{m} \rightarrow s$. Subnets are useful because, in particular, a topological space $X$ is compact if and only if each net in $X$ has a subnet which converges to some point in $X$ (see Kelley (1955, Theorem 5.2, p. 136)).

Nets and subnets play in topological spaces an analog role to sequences and subsequences in metric spaces. For instance, we have that a point $s$ belongs to the closure of a subset $A$ of a topological space if and only if there is a net converging to $s$ (see Kelley (1955, Theorem 2.2, p. 66)). Furthermore, a function $f: X \rightarrow Y$, where $X$ and $Y$ are topological spaces, is continuous if and only if $\left\{f\left(x_{j}\right)\right\}_{j \in J}$ converges to $f(x)$ for all $x \in X$ and all nets $\left\{x_{j}\right\}_{j \in J}$ converging to $x$ (Kelley (1955, Theorem 3.1, p. 86)).

Let $X$ and $Y$ be topological spaces. A correspondence $\varphi: X \rightrightarrows Y$ is upper hemicontinuous if for all $x \in X$, all nets $\left\{x_{j}\right\}_{j \in J}$ in $X$ converging to $x$ and all open sets $G$ in $Y$ with $\varphi(x) \subseteq G$, there exists $j_{0} \in J$ such that $\varphi\left(x_{j}\right) \subseteq G$ for all $j \succeq j_{0}$ (see Klein and Thompson (1984, Theorem 7.1.4, p. 74)).

A correspondence $\varphi: X \rightrightarrows Y$ is lower hemicontinuity if for all $x \in X$, all nets $\left\{x_{j}\right\}_{j \in J}$ in $X$ converging to $x$ and all open sets $G$ in $Y$ with $\varphi(x) \cap G \neq \emptyset$, there exists $j_{0} \in J$ such that $\varphi\left(x_{j}\right) \cap G \neq \emptyset$ for all $j \succeq j_{0}$ (see Klein and Thompson (1984, Theorem 7.1.7, p. 75)).

The following result concerns lower hemicontinuous correspondences $\varphi$ : $X \rightrightarrows Y$. It is well known that if $X$ and $Y$ are metric spaces, $x$ belongs to $X, y$ belongs to $\varphi(x)$ and $\left\{x_{n}\right\}_{n=1}^{\infty} \subseteq X$ converges to $x$ then, there exists a corresponding sequence $\left\{y_{n}\right\}_{n=1}^{\infty}$ converging to $y$ and with the property that $y_{n} \in \varphi\left(x_{n}\right)$ for all $n \in \mathbb{N}$ (see Hildenbrand and Kirman (1988, Theorem AIII.2, p. 271)). Lemma 5 is the analog of this result when both $X$ and $Y$ are topological spaces.

Lemma 5 Let $X$ and $Y$ be topological spaces and $\varphi$ a lower hemicontinuous correspondence from $X$ to $Y$. Let $\left\{x_{j}\right\}_{j \in J}$ be a net in $X$ converging to $x \in X$ and $y \in \varphi(x)$.

Then, there exists a subnet $\left\{\tilde{x}_{m}\right\}_{m \in M}$ of $\left\{x_{j}\right\}_{j \in J}$ and a corresponding net $\left\{y_{m}\right\}_{m \in M}$ such that $y_{m} \in \varphi\left(s_{m}\right)$ for all $m \in M$ and $y_{m} \rightarrow y$. 
Proof. Let $\mathcal{V}_{y}$ be the set of all open neighborhoods of $y$, directed by $\subseteq$. Since $\varphi$ is lower hemi-continuous, then for all $V \in \mathcal{V}_{y}$ there exists $j_{V} \in J$ such that $\varphi\left(x_{j}\right) \cap V \neq \emptyset$ for all $j \succeq j_{V}$.

Define a function $N: \mathcal{V}_{y} \times J \rightarrow J$ by choosing, for all $V \in \mathcal{V}_{y}$ and $j \in J$, an element $N(V, j) \in J$ satisfying $N(V, j) \succeq j_{V}$ and $N(V, j) \succeq j$. We direct $\mathcal{V}_{y} \times J$ by defining $(V, j) \succeq\left(V^{\prime}, j^{\prime}\right)$ if and only if $V \subseteq V^{\prime}$ and $j \succeq j^{\prime}$. Define $\tilde{x}_{V, j}=x_{N(V, j)}$ for all $(V, j) \in \mathcal{V}_{y} \times J$.

It is clear that $N$ is admissible. Indeed, if $j \in J$, we can consider $(X, j) \in$ $\mathcal{V}_{y} \times J$, showing that $\left(V^{\prime}, j^{\prime}\right) \succeq(X, j)$ implies that $N\left(V^{\prime}, j^{\prime}\right) \succeq j^{\prime} \succeq j$.

Let $V \in \mathcal{V}_{y}$ and $j \in J$. Note that $\varphi\left(\tilde{x}_{V, j}\right) \cap V \neq \emptyset$ since $\tilde{x}_{V, j}=x_{N(V, j)}$ and $N(V, j) \succeq j_{V}$. Hence, choose $y_{V, j} \in \varphi\left(\tilde{x}_{V, j}\right)$.

It remains to show that $y_{V, j} \rightarrow y$. Let $V \in \mathcal{V}_{y}$. We can take an arbitrary $j_{0} \in J$, and we have that $y_{V^{\prime}, j^{\prime}} \in V^{\prime} \subseteq V$ for all $\left(V^{\prime}, j^{\prime}\right) \succeq\left(V, j_{0}\right)$.

Thus, let $M=\mathcal{V}_{y} \times J$ and for all $m \in M$, let $\tilde{x}_{m}=\tilde{x}_{V, j}=x_{N(V, j)}$, where $m=(V, j)$.

\section{References}

Börgers, T. (1989): "Perfect Equilibrium Histories of Finite and Infinite Horizon Games," Journal of Economic Theory, 47, 218-227.

(1991): "Upper Hemicontinuity of the Correspondence of SubgamePerfect Equilibrium Outcomes," Journal of Mathematical Economics, 20, 89-106.

HARRIS, C. (1985a): "A Characterizarion of the Perfect Equilibria of Infinite Horizon Games," Journal of Economic Theory, 37, 99-125.

(1985b): "Existence and Characterization of Perfect Equilibrium in Games of Perfect Information," Econometrica, 53, 613-628.

Hellwig, M., and W. Leininger (1987): "On the Existence of SubgamePerfect Equilibrium in Infinite-Action Games of Perfect Information," Journal of Economic Theory, 43, 55-75.

Hellwig, M., W. Leininger, P. Reny, and A. Robson (1990): "Subgame Perfect Equilibrium in Continuous Games of Perfect Information: An Elementary Approach to Existence and Approximation by Discrete Games," Journal of Economic Theory, 52, 406-422. 
Hildenbrand, W., ANd A. Kirman (1988): Equilibrium Analysis. North Holland, Amsterdam.

Kelley, J. (1955): General Topology. Springer, New York.

Klein, E., And A. Thompson (1984): Theory of Correspondences. Wiley, New York.

Wheeden, R., And A. Zygmund (1977): Measure and Integral: An Introduction to Real Analysis. Dekker, New York. 